Acta vet. scand. $1970,11,487-490$.

Brief Communication

\title{
A NEW PORCINE MYCOPLASMA SPECIES: MYCOPLASMA SUIDANIAE
}

In an investigation of the mycoplasma flora in lungs of Danish pigs, 12 strains of an alkali-producing agent were isolated from lungs with catarrhal pneumonia. The pigs in question originated from 9 different farms. Details concerning media and technique are given in a previous paper (Friis 1971).

By the growth inhibition test, the 12 strains were shown to be closely related. One strain (M60) was chosen as a representative of the group and submitted to a number of tests in order to identify it as a mycoplasma.

Strain M60 was originally isolated in medium-FF (Friis 1971) and later adapted to a variant of this medium, in which glucose and $\mathrm{NaHCO}_{3}$ were omitted and $0.2 \%$ arginine added. Adjustment of $\mathrm{pH}$ to 7.3. In this fluid medium growth was obtained after 24 hrs., evidenced by a color-shift of the phenol-red indicator from yellowish to bluish ( $\mathrm{pH} 8.25)$. Un-inoculated control tubes showed a $\mathrm{pH}$ of 7.55. In the terminal phase of growth the fluid became turbid with a distinct grease-like layer on the surface (film and spot). In medium without arginine growth was obtained after 48 hrs. with a minimum of color-shift; the $\mathrm{pH}$ was 7.65 in inoculated tubes, as against 7.55 in un-inoculated control tubes. Thus, the capacity of M60 to produce alkali by degradation of arginine was demonstrated.

On solid medium, colonies of the typical "fried-egg" appearance were seen after 2 days. Their size ranged from 200 to $500 \mu$, and on prolonged incubation a crystalline structure developed round the colonies (film and spot).

Serum-free medium would not support growth of M60. Omission of bacteriostatics did not result in reversion to a parent bacterium, which indicated that $\mathrm{M60}$ is not an L-phase variant. Moreover, M60 was completely unaffected by penicillin at a concentration of $10 \mathrm{mg}$ per $\mathrm{ml}$, but highly sensitive to tetracyclin, the minimal effective dose being $0.1 \gamma$ per $\mathrm{ml}$. 
From filtration studies the size of the minimal reproductive unit was estimated at about $0.2 \mu$. By phase-contrast microscopy of broth cultures (Fig. 1) pleomorphism and mycelial growth of M 60 were noted. Organisms from fluid medium could be stained by the Giemsa-method (Fig. 2) but not by the usual bacteriological staining procedures.

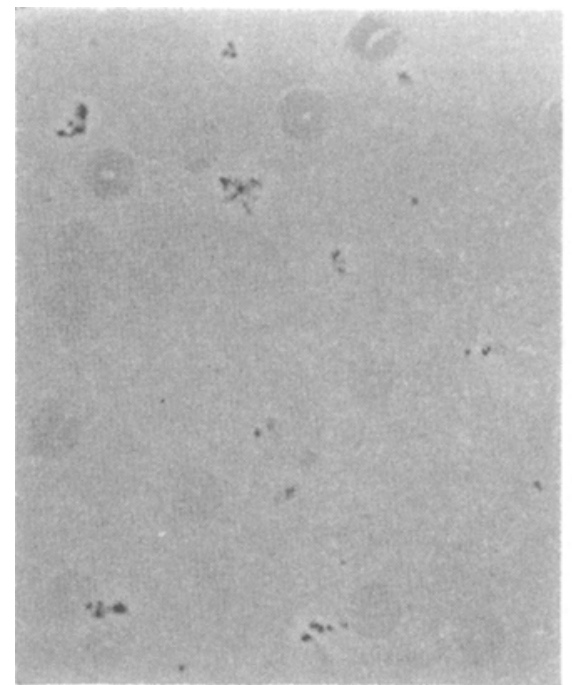

F i g u r e 1. Phase-contrast. $\times 1000$.

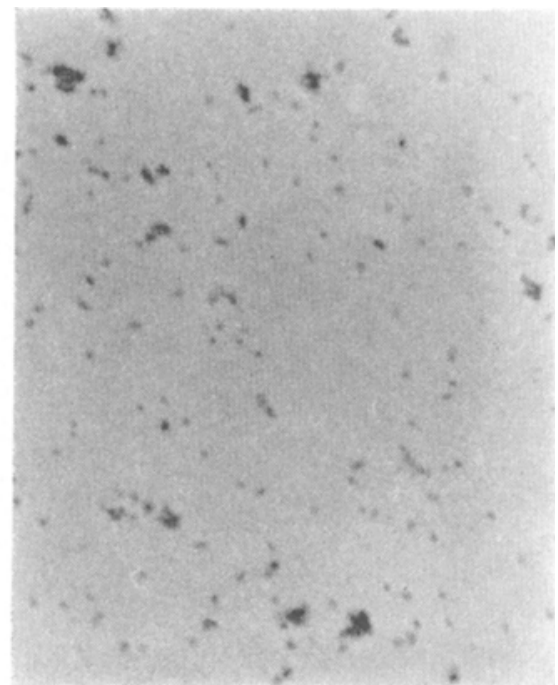

F i g u r e 2. Giemsa staining. $\times 1000$.

Studies on the metabolic activities of M60 showed that only arginine was degraded, whereas urea, glucose, mannose, lactose, saccharose, maltose, arabinose, rhamnose, trehalose, xylose, mannitol, sorbitol, dulcitol, inositol, and salicin were not. Attempts to demonstrate reduction of tetrazolium and phosphatase activity both gave negative results.

Serologic comparison was performed, M60 antiserum being used in the growth and metabolic inhibition tests against a number of mycoplasma species. In no case was any relationship demonstrable between M60 and recognized species, for which reason it was concluded that $\mathrm{M} 60$ is a true, serologically specific mycoplasma.

For the primary isolation of this mycoplasma species, a number of different media were tried. A medium composed as follows was found to be most suitable, and definitely better than the 
earlier described medium-E: Double-distilled water, $380 \mathrm{ml}$; PPLO broth w/o CV (Difco), 8.0 g; yeast extract (Difco), $0.3 \mathrm{~g}$; mucin bacteriological (Difco), $0.1 \mathrm{~g}$. After autoclaving and cooling, add freshly prepared yeast extract, $20 \mathrm{ml} ; 0.5 \%$ phenol-red solution, $1.5 \mathrm{ml}$; penicillin, 100,000 i.u.; $5 \%$ thallium acetate solution, $0.9 \mathrm{ml} ; 8 \%$ arginine solution, $12 \mathrm{ml}$; horse serum, 50 $\mathrm{ml}$; and pig serum, $50 \mathrm{ml}$. Adjust $\mathrm{pH}$ to 7.25 . For further details concerning preparation of media, see Friis 1971.

Another strain (M152) was used for preliminary pathogenicity studies of 7 10-week-old pigs. All the pigs were infected by aerosol and by intra-articular injection in 3 major joints. They were killed on, respectively, day $9,12,13,15,22,27$, and 41 p.i. None of the pigs showed any clinical or pathological evidence of respiratory disease, nor was the agent recovered from the lungs, except in the case of the pig killed on day 9 p.i. The joints revealed no definite clinical signs of disease, although 2 pigs showed slightly stiff movements. At necropsy the synovial fluid was in some cases found to be increased and whitish or brownish discolored. On microscopy a proliferation of lympho-reticular cells was found. In most cases the agent could be recovered from the inoculated joints.

The relationship of strain $\mathbf{M 6 0}$ to the alkalinizing porcine mycoplasmas reported on by Moore et al. (1966) and Pillai et al. (1967) has not been examined, because these strains were not available on request. The alkalinizing isolates (B6) from pigs described by Dinter et al. (1965) were shown to be strains of M. iners (Taylor-Robinson \& Dinter 1968). M60, however, did not show any relation to $M$. iners in the serologic tests used in this study.

On the basis of the data outlined above it is therefore concluded that a new porcine species of mycoplasma belonging to the arginine-metabolizing group has been discovered. It is proposed that this new species be given the name Mycoplasma suidaniae.

Niels F. Friis

The State Veterinary Serum Laboratory, Copenhagen, Denmark.

\section{REFERENCES}

Dinter, Z., D. Danielsson \& $K$. Bakos: Differentiation of porcine mycoplasma strains. J. gen. Microbiol. 1965, 41, 77-84. 
Friis, N. F.: Mycoplasmas cultivated from the respiratory tract of Danish pigs. Acta vet. scand. 1971, 12. In press.

Moore, R. W., H. E. Redmond \& C. W. Livingston jr.: Pathologic and serologic characteristics of a mycoplasma causing arthritis in swine. Amer. J. vet. Res. 1966, 27, 1649-1656.

Pillai, Carolyn, G. T. Woods, H. E. Rhoades \& J. Simon: Results of exposure of colostrum-deprived pigs to a strain of mycoplasma sp. isolated from a swine lung. Illinois Vet. 1967, 10, 12-14.

Taylor-Robinson, D. \& Z. Dinter: Unexpected serotypes of mycoplasmas isolated from pigs. J. gen. Microbiol. 1968, 53, 221-229.

(Received August 13, 1970). 